Reprad. Nutr. Dévelop., 1980, 20 (4 B), 1317-1323.

\title{
Action de la puromycine sur l'absorption intestinale des lipides chez le rat mâle et femelle
}

\author{
par A. BERNARD, A. DUTERTRE, Hélène CARLIER \\ avec la collaboration technique de Marie-France GIRARDIER \\ Laboratoire de Physiologie de la Nutrition L. A. 273 \\ ENS.BANA Campus Montmuzard, 21100 Dijon, France.
}

Summary. Puromycin action on intestinal lipid absorption in male and female rats.

The effect of puromycin on absorption balance and mucosal lipid distribution was studied during intestinal absorption of ${ }^{14} \mathrm{C}$ oleic acid and ${ }^{3} \mathrm{H}$ palmitic acid, using the isolated intestinal loop of male and female rats in situ.

In puromycin-treated animals, mucosal lipid uplake was reduced and mucosal lipid content increased, particularly with oleic acid and in male rats.

The phospholipids were significantly reduced. As revealed by mucosal accumulation of free fatty acids, esterification was particularly inhibited with oleic acid and in female rats.

These results might explain why portal blood radioactivity was higher in treated rats than in the controls.

Puromycin effect occurred at different stages of lipid absorption and varied with sex.

\section{Introduction.}

Dans le cadre de notre travail sur l'étude de la participation des organites de l'entérocyłe à l'absorption des lipides, nous recherchons un inhibiteur de la synthèse protéique susceptible de bloquer une étape particulière de l'absorption des acides gras à longue chaîne. Ceci afin d'étudier les modifications morphologiques provoquées dans la cellule absorbante et d'essayer d'en déduire le rôle des différents organites subcellulaires dans l'absorption des acides gras. Pour choisir l'inhibiteur susceptible de bloquer soit l'estérification, soit l'enrobage des triglycérides resynthétisés au sein de l'entérocyłe, il nous faut, dans un premier temps, connaître les répercussions de son action sur l'estérification au niveau de la muqueuse ef les bilans d'absorption par rapport aux conditions normales.

Parmi ces inhibiteurs, la puromycine, selon certains auteurs, n'inhiberait pas l'estérification des acides gras à longue chaîne (Sabesin et Isselbacher, 1965 ; O'Doherty, Yousef et Kuksis, 1973 ; O’Doherty et Kuksis, 1974 ef 1975). Néanmoins, la quantité des triglycérides lymphatiques est diminuée (Kayden et Medick, 1969 ; Redgrave et Zilversmit, 1969) ce qui est confirmé par une diminution de la sécrétion des chylomicrons (Yousef et Kuksis, 1972). D'autres travaux montrent au contraire une 
inhibition de l'estérification (Redgrave et Zilversmit, 1969 ; Vahouny et al., 1977) et surtout une sensibilité différente des animaux selon le sexe (Vahouny ef al., 1977).

Pour essayer de voir ce qu'il en était réellement, nous avons étudié in vivo chez le Rat mâle et femelle, l'action de la puromycine sur le prélèvement des lipides, leur estérification et leur disparition, au niveau de l'entérocyte, en utilisant en même temps deux acides gras à longue chaîne, l'un saturé, l'acide palmitique, marqué au tritium, l'autre insaturé, l'acide oléique marqué au carbone 14.

\section{Matériel et méthodes.}

\section{Matériel.}

Les expériences sont réalisées sur des rats mâles et femelles adultes de souche Wistar, pesant de 180 à $220 \mathrm{~g}$.

Le suc pancréatique est dérivé par fistulation du canal pancréato-biliaire à $3 \mathrm{~mm}$ environ de son abouchement dans le duodénum (cathéter polyéthylène Biotrol $n^{\circ} 1$ ). La bile est réintroduite dans l'intestin à l'aide d'un second cathéter reliant la partie supérieure du canal pancréato-biliaire, juste au-dessus du tissu pancréatique, à l'endroit d'arrivée du canal dans le duodénum. Le rat opéré, placé dans une cage à contention, a libre accès à l'eau de boisson, mais ne reçoit aucune nourriture.

Vingt-quatre heures plus tard, sous légère anesthésie à l'éther, on isole in situ par la pose de deux canules (Collin-Gentille no 18) une anse intestinale de $15 \mathrm{~cm}$ de longueur, située 2 à $3 \mathrm{~cm}$ après l'abouchement du canal pancréato-biliaire. On rince l'anse intestinale avec $5 \mathrm{ml}$ de Ringer pour mammifères à $37^{\circ} \mathrm{C}$. On introduit l'émulsion lipidique maintenue da $37^{\circ} \mathrm{C}$.

Celle-ci est composée de $30 \mu \mathrm{mol}$. de monopalmitine, $30 \mu \mathrm{mol}$. d'acide palmitique tritié en 9-10 $(25 \mu \mathrm{Ci})$ et de $30 \mu \mathrm{mol}$. d'acide oléique marqué au ${ }^{14} \mathrm{C}$ sur le carboxyle $(10 \mu \mathrm{Ci})^{*}$, auxquelles on ajoute $1,5 \mathrm{ml}$ de bile d'un rat donneur.

Les animaux reçoivent la puromycine (Puromycine dihydrochlorid crystallised, Boehringer-Mannheim) par voie intrapéritonéale $(10 \mathrm{mg}$ au total contenus dans $9 \mathrm{ml}$ de tampon phosphate $0,04 \mathrm{M}, \mathrm{pH} 7,4$ ) en quatre injections de $2 \mathrm{ml}$ espacées d'1 h, $4 \mathrm{~h}$ avant l'expérience, la dernière injection de $1 \mathrm{ml}$ précédant l'infusion du milieu lipidique. Les rats témoins reçoivent, dans les mêmes conditions, une même quantité de soluté sans puromycine.

\section{Techniques.}

Trente minutes après infusion de l'émulsion lipidique, on extrait les lipides du contenu intestinal, de la muqueuse, du reste de l'émulsion lipidique non introduite ainsi que de quelques échantillons de sang de la veine porte (recueillie à l'aide d'un cathéter entre la $28 \mathrm{e}$ et la $30 \mathrm{e} \mathrm{min}$ ) par un mélange de diméthoxyméthane-méthanol $4 / 1 \mathrm{~V} / \mathrm{V}$ (Delsal, 1944). On sèche les extraits à l'alcool absolu et on reprend par du chloroforme. Les lipides sont séparés par chromatographie sur couches minces de gel

* La pureté chimique et radioactive des acides gras marqués (CEA, Saclay, France) a été vérifiée par spectrométrie par scintillation liquide des acides gras séparés par chromatographie gaz-liquide. 


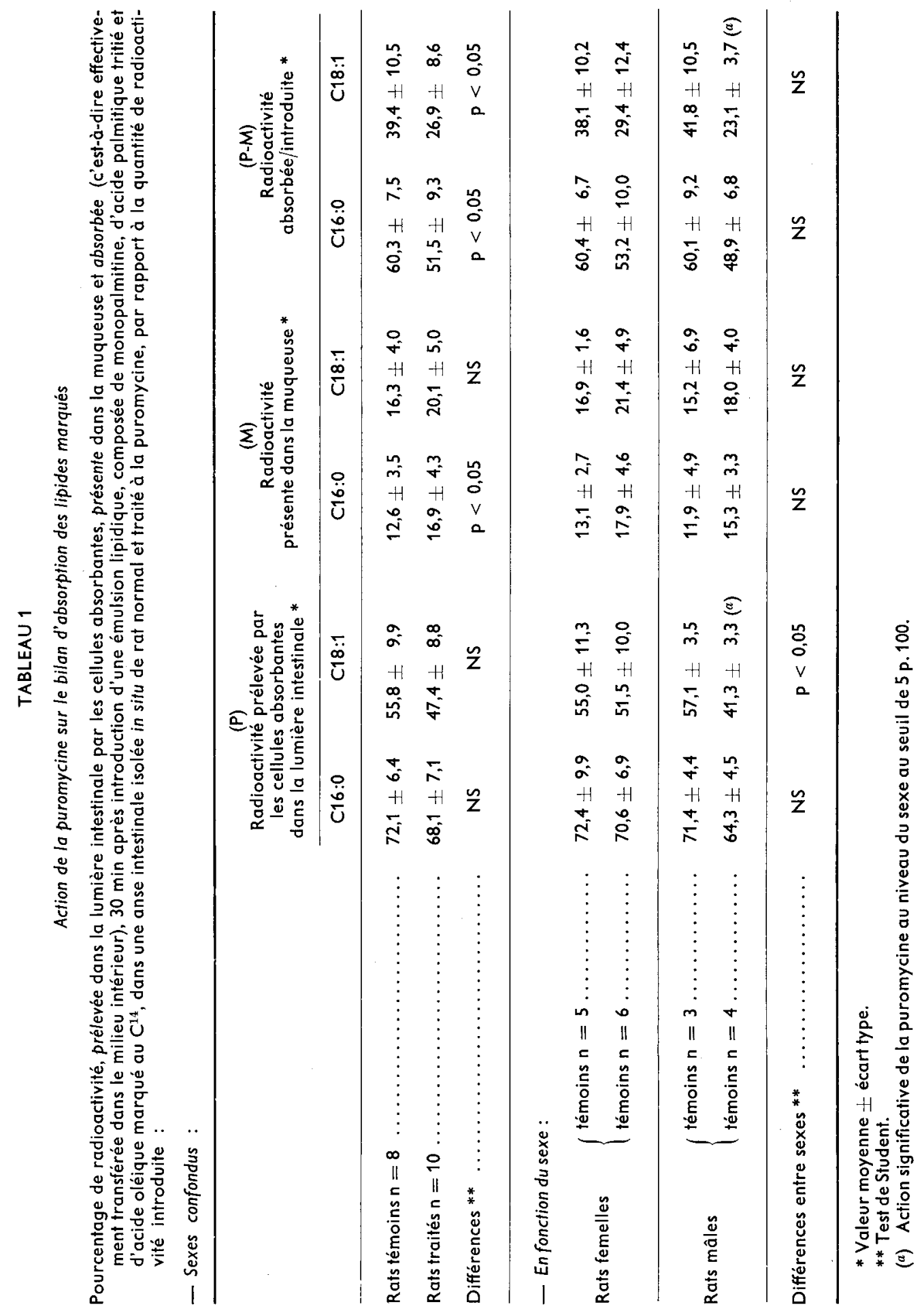


de silice de $500 \mu \mathrm{m}$ d'épaisseur selon la technique de Stahl et al. (1956) avec le milieu de développement suivant : éther de pétrole, éther éthylique, acide acétique, méthanol : $90 / 20 / 2 / 3 \mathrm{~V} / \mathrm{V} / \mathrm{V} / \mathrm{V}$. Après migration, on utilise les vapeurs d'iode pour révéler les lipides sur les plaques. L'acide silicique sur lequel est adsorbé chaque constituant, est prélevé par grattage et introduit directement dans des pots à scintillation contenant $0,5 \mathrm{ml}$ de méthanol pour élver les lipides. Après 12 heures, on ajoute $5 \mathrm{ml}$ de liquide scintillant (4 g de 2-5, diphényloxazole et $100 \mathrm{mg}$ de 1,4-bis 2 (4-méthyl-5-phényloxazolyl)-benzène pour 1 I de toluène Merck) et on mesure la radioactivité à l'aide d'un compteur à scintillation liquide Packard Prias PLD TRI-CARB. Les rendements de comptage sont déterminés par l'emploi d'un standard externe, la correction automatique du quenching se faisant elle par l'intermédiaire d'un standard interne.

Par rapport à la radioactivité introduite prise égale à 100 pour chacune des molécules marquées, on calcule le pourcentage de radioactivité :

- prélevée dans la lumière intestinale $(P)$,

- présente dans la muqueuse $(M)$, et

- absorbée dans le milieu intérieur (P-M).

\section{Résultats.}

1. Bilan d'absorption (tabl. 1). - Si l'on considère l'ensemble des rats mâles ef femelles (sexes confondus), la puromycine entraîne une diminution des acides gras prélevés par la muqueuse mais celle-ci n'est pas statistiquement significative. Les rats mâles paraissent plus sensibles à l'action de la puromycine que les rats femelles, notamment pour l'absorption de l'acide oléique pour lequel la différence entre sexes est significative.

L'inhibition de la synthèse protéique s'accompagne d'une légère surcharge des lipides marqués de la muqueuse, du même ordre pour les deux sexes, significative pour l'acide palmitique et les sexes confondus.

Le transfert des lipides marqués est significativement diminué par action de la puromycine. Les rats mâles présentent également une plus grande sensibilité que les rats femelles, sans cependant que les différences soient significatives.

2. Répartition de la radioactivité dans la muqueuse intestinale (tabl. 2). - L'inhibition de l'estérification s'accompagne d'une augmentation relative très significative des acides gras libres marqués pour les animaux des deux sexes, mais nettement plus significative chez les rats femelles avec l'acide oléique. Parallèlement, la diminution des phospholipides marqués est significative et c'est essentiellement la fraction lécithines qui est affectée. En effet, les lécithines qui représentent de 2,3 à 3,5 p. 100 des phospholipides chez les rats témoins, n'en représentent plus que 0,5 à 1,1 p. 100 chez les traités.

3. Radioactivité du sang portal. - Des prélèvements du sang de la veine porte hépatique, effectués entre la $28^{\mathrm{e}}$ et la $30^{\circ} \mathrm{min}$ de l'absorption révèlent une radioactivité nettement plus importante du sang chez les animaux traités comparés aux animaux témoins $(+32$ à +34 p. 100 pour les lipides marqués respectivement à l'acide palmitique et oléique chez les rats femelles et +147 ef +325 p. 100 pour les acides palmitique et oléique respectivement pour les rats mâles). 


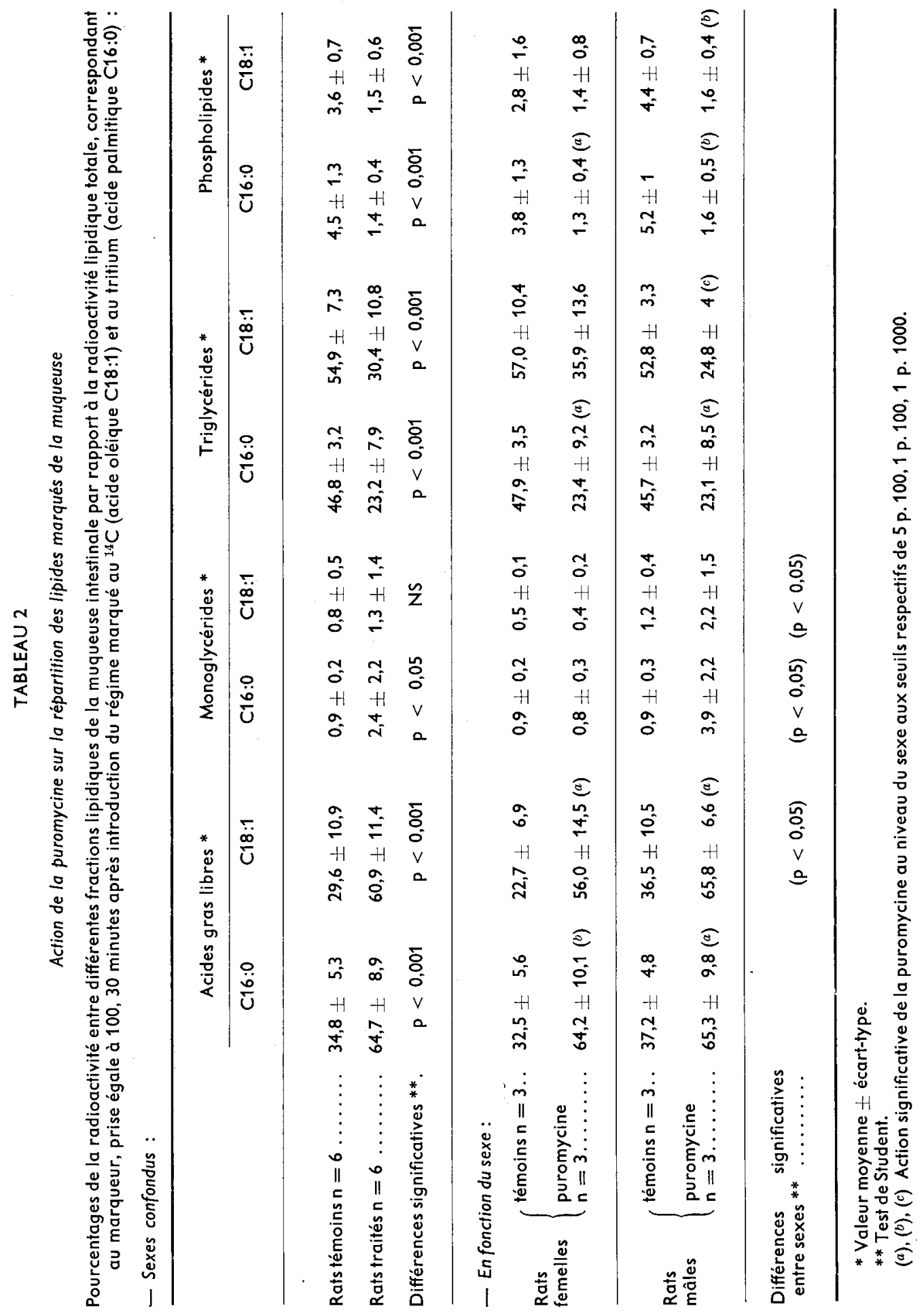




\section{Discussion.}

La légère surcharge en lipides marqués que nous constatons au niveau de la muqueuse, est en accord avec les observations histologiques de Sabesin ef Isselbacher (1965) et ultrastructurales de Friedman et Cardell (1972). En revanche, les premiers auteurs ne relevaient pas de modifications au niveau de l'estérification chez les rats femelles traités par rapport aux témoins, ainsi le $C^{\mathbf{1 4}^{4}}$ palmitate estérifié passait de $59 \mathrm{~m} \mu \mathrm{mol} . / \mathrm{mg}$ chez les rats témoins à $52 \mathrm{~m} \mu \mathrm{mol} / \mathrm{mg}$ chez les traités. Nos résultats montrent au contraire des différences nettes pour les animaux des deux sexes. Cependant, d'autres expériences réalisées sur cellules isolées ou sur microsomes d'entérocyłes de rats femelles confirmaient que la synthèse des triglycérides n'était pas affectée par l'action de la puromycine (O'Doherty, Yousef ef Kuksis, 1973 ; O'Doherty et Kuksis, 1974, 1975). Selon les résultats de ces différents travaux, pour lesquels l'estérification des acides gras ne semblait pas compromise, la puromycine se révélait être un outil précieux pour étudier le problème même de la formation, de l'organisation et de l'exocytose des chylomicrons à partir des triglycérides, en supposant que leur hydrolyse n'ait pas lieu (Negrel, Serrero et Aihlaud, 1977).

La diminution très netfe des phospholipides marqués, observée après action de la puromycine, pour les animaux des deux sexes, est en accord avec les travaux biochimiques de divers auteurs réalisés chez le rał femelle (O’Doherty, Yousef et Kuksis, 1973 ; O'Doherty et Kuksis, 1974, 1975), et en accord également avec les résultats de Vahouny et al. (1977) obtenus pour les animaux des deux sexes. Cette action sur la formation des phospholipides peut en partie s'expliquer par la désorganisation du réticulum endoplasmique granuleux observée en microscopie électronique par Friedman et Cardell (1972) chez le rat mâle traité à la puromycine.

Tous sexes confondus, l'accumulation relative très nette des acides gras libres marqués dans la muqueuse que nous observons, confirme les observations de Vahouny ef al. (1977), avec toutefois cette différence que l'accumulation est plus sensible avec l'acide oléique dans nos conditions expérimentales chez les rats femelles, alors que pour ces auteurs, elle apparaissait seulement significative chez les rats mâles. La diminution parallèle des triglycérides marqués que nous observons rejoint les résultats de Redgrave et Zilversmit (1969) observés chez le rat femelle, nos résultats étant cependant plus nets. Vahouny et al. (1977) rapportent ce phénomène seulement chez les rats mâles et, bien que significatif, beaucoup plus discret. L'action de la puromycine, en fonction du sexe de l'animal n'apparaît donc pas toujours aussi clairement. Il est vrai que nos conditions expérimentales ne sont pas les mêmes que celles de Vahouny ef al. (1977), puisque nous infusons notre émulsion lipidique dans la lumière intestinale, et supprimons ainsi l'effet de la puromycine sur l'éclusage gastrique.

Ainsi dans nos conditions expérimentales, in vivo, la puromycine agit en amont de l'intégration des triglycérides dans la structure des chylomicrons. Cette action explique la libération moindre de chylomicrons par l'entérocyte (Yousef et Kuksis, 1972) ou de lymphe (Kayden et Medick, 1969 ; Vahouny et al., 1977). A cet égard, les résultats observés sur le sang de la veine porte, bien que très ponctuels, appuient l'hypothèse selon laquelle la voie portale devient la voie d'absorption, en cas de défaillance du processus d'estérification (Huyn, Vahouny et Treadwell, 1967), comme le suggéraient d'ailleurs Kayden et Medick (1969). 
Pour relier l'évolution des organites subcellulaires de l'entérocyte aux différents processus biochimiques et membranaires qui accompagnent l'absorption intestinale des acides gras à longue chaîne, la recherche d'un inhibiteur de la synthèse protéique, ayant une action moins irradiante reste posé. Le rat mâle apparaît plus sensible sur le plan bilan d'absorption et le rat femelle sur celui de l'inhibition de l'estérification, et généralement l'action de la puromycine est plus nette avec l'acide oléique. Toutefois, à la lumière des résultats obtenus ici, les observations biochimiques ef ultrastructurales réalisées avec la cycloheximide ou ses dérivés (Bernard et Carlier, 1980 ; Bernard, Carlier et Caselli, 1980), nous font momentanément préférer ces derniers.

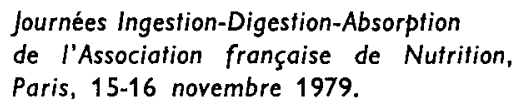

Remerciements. - Nous remercions M. F. Sauvageot, chargé de recherches à l'ENS. BANA, pour le traitement statistique des résultats, et nous remercions vivement $M$. le Professeur J. Bezard, directeur du laboratoire, pour les conseils prodigués lors de ce iravail.

\section{Références}

BERNARD A., CARLIER H., 1980. Action d'un inhibiteur de la synthèse protéique, l'actidione-cycloheximide, sur l'ultrastructure de l'entérocyte de rał à jeun. J. Physiol. Paris, 76, 71-77.

BERNARD A., CARLIER H., CASELLI C., 1980. Ełude biochimique et ultrastructurale de I'action de l'actidione-cycloheximide sur l'absorption intestinale des lipides chez le rat. J. Physiol. Paris (sous presse).

DELSAL J. L., 1944. Nouveau procédé d'extraction des lipides du sérum par le méthylal. Application aux microdosages du cholestérol total, des phosphoaminolipides et des protéines. Bull. Soc. Chim. biol., 26, 99-105.

FRIEDMAN H. I., CARDELL R. R. Jr, 1972. Effects of puromycin on the structure of rat intestinal epithelial cells during fat absorption. J. Cell Biol., 52, 15-40.

HUYN S. A., VAHOUNY G. V., TREADWELL C. R., 1967. Portal absorption of fatty acids in lymphand portal vein-cannulated rats. Biochim. biophys. Acta, 137, 296-305.

KAYDEN H. J., MEDICK M., 1969. The absorption and metabolism of short and long chain fatty acids in puromycin-treated rats. Biochim. biophys. Acta, 176, 37-43.

NEGREL R., SERRERO G., AILHAUD G., 1977. Lipolytic activities against trioctanoin and monoolein in rat intestinal mucosa. Biochimie, 59, 433-438.

O'DOHERTY P. J. A., KUKSIS A., 1974. Differential effect of puromycin on triacylglycerol and phosphatidylcholine synthesis in rat mucosal microsomes. Con. J. Biochem., 52, 170-175.

O'DOHERTY P. J. A., KUKSIS A., 1975. Effect of puromycin in vitro on protein and glycerolipid biosynthesis in isolated epithelial cells on rat intestine. Int. J. Biochem., 6, 435-441.

O'DOHERTY P. J. A., YOUSEF I. M., KUKSIS A., 1973. Effect of puromycin on protein and glycerolipid biosynthesis in isolated mucosal cells. Arch. Biochem. biophys., 156, 586-594.

REDGRAVE T. G., ZILVERSMIT D. B., 1969. Does puromycin block release of chylomicrons from the intestine? Am. J. Physiol., 217, 336-340.

SABESIN S. M., ISSELBACHER K. J., 1965. Protein synthesis inhibition : mechanism for the production of impaired fat absorption. Science, 147, 1149-1151.

STAHL E., SHROTER G., KRAFT G., RENZ R., 1956. Thin layer chromatography (the method affecting factors, and a few examples of application). Pharmazie, 11, 633-637.

VAHOUNY G. V., ITO M., BLENDERMANN E. M., GALLO L. L., TREADWELL C. R., 1977. PUROmycin inhibition of cholesterol absorption in the rat. J. Lipid Res., 18, 745-752.

YOUSEF I. M., KUKSIS A., 1972. Release of chylomicrons by isolated cells of rat intestinal mucosa. Lipids, 7, 380-386. 